\title{
Lifting dual tree complex wavelets transform
}

\author{
Hilal Naimi, Amelbahahouda Adamou-Mitiche, Lahcène Mitiche \\ Laboratoire de Modélisation Simulation et Optimisation des Systèmes Complexes Reels, Université de Djelfa, Algeria
}

\begin{tabular}{l} 
Article Info \\
\hline Article history: \\
Received Aug 8, 2020 \\
Revised Mar 3, 2021 \\
Accepted Apr 19, 2021 \\
\hline Keywords: \\
Dual tree complex wavelets \\
transform \\
Image denoising \\
Lifting wavelet transform \\
Wavelet shrinkage
\end{tabular}

Corresponding Author:

Hilal Naimi

Faculty of Science and Technology

University of Ziane Achour Djelfa

Moudjbara road, Djelfa 17000, Algeria

Email: h.naimi@mail.univ-djelfa.dz

\begin{abstract}
We describe the lifting dual tree complex wavelet transform (LDTCWT), a type of lifting wavelets remodeling that produce complex coefficients by employing a dual tree of lifting wavelets filters to get its real part and imaginary part. Permits the remodel to produce approximate shift invariance, directionally selective filters and reduces the computation time (properties lacking within the classical wavelets transform). We describe a way to estimate the accuracy of this approximation and style appropriate filters to attain this. These benefits are often exploited among applications like denoising, segmentation, image fusion and compression. The results of applications shrinkage denoising demonstrate objective and subjective enhancements over the dual tree complex wavelet transform (DTCWT). The results of the shrinkage denoising example application indicate empirical and subjective enhancements over the DTCWT. The new transform with the DTCWT provide a trade-off between denoising computational competence of performance, and memory necessities. We tend to use the PSNR (peak signal to noise ratio) alongside the structural similarity index measure (SSIM) and the SSIM map to estimate denoised image quality.
\end{abstract}

This is an open access article under the CC BY-SA license.

\section{INTRODUCTION}

The discrete wavelet transforms (DWT) is with success applied to numerous issues in signal and image processing. Data compression, data interpretation, data hiding, audio signal processing, motion tracking, and machine learning. It has been recognized that the DWT suffers from shift variance [1], [2] and high computational competence, and memory necessities. Dual tree complex wavelet transform and lifting wavelet transform (LWT) are developed to beat these drawbacks. LWT was started as a way to boost a given DWT to get specific properties. Later it became an active algorithm to calculate any wavelet transforms as a sequence of easy lifting steps. Digital signals are usually a sequence of integer numbers, whereas wavelet transforms end in floating purpose numbers. For an efficient reversible implementation, it's of nice importance to possess a transform algorithm that converts integers to integers [3], [4]. Fortunately, a lifting step may be changed to work on integers, whereas preserving the reversibility [3]. Thus, the lifting scheme became a way to implement reversible integer wavelet transforms. More recently, the improved directional selectivity and complex subbands (DTCWT) has offered a more compact representation in two and higher dimensions, while providing near-shift invariance and directionally selective [5]. This paper proposes lifting scheme form of the DTCWT which combines the benefits of the LWT and the DTCWT. 


\section{LIFTING SCHEME}

The basic idea back the lifting scheme is to begin with an initial wavelet named the "Lazy Wavelet". There is no function connected with these lifting wavelets, except that it has the properties of a wavelet [6], [7]. The lifting scheme then tries to structure new wavelet by adding new basis functions. The lifting scheme then ameliorates the properties of the builder wavelet by finding a close liaison between the low and high frequency components. This is the revelation back the name "lifting scheme". Daubechies and Sweldon [8], [9] proved that a new construction of wavelet transform can be builder from any orthogonal and biorthogonal filters by using factorization of a polyphase matrix. So the lifting scheme starts with a well-known collection of filters, say (h, g), and the filters are section into even and odd.

$$
P(z)=\left(\begin{array}{ll}
h_{e} & g_{e} \\
h_{o} & g_{o}
\end{array}\right)
$$

The polyphase matrix has more than one factorization. The quotient can be chosen in a variety of ways, and the division can then check a completely different set of lifting coefficients [10]. The goal of the factorization is to exemplify the polyphase matrix as a collection of upper and lower triangular matrices. This can be written as (2).

$$
P(z)=\left(\begin{array}{cc}
K & 1 \\
1 & 1 / K
\end{array}\right) \prod_{i=1}^{m}\left(\begin{array}{cc}
1 & S_{i}(z) \\
0 & 1
\end{array}\right)\left(\begin{array}{cc}
1 & 0 \\
t_{i}(z) & 1
\end{array}\right)
$$

where, $\mathrm{K}$ is a non-zero constant and the Laurent polynomials.

$S_{i}(z)$ and $t_{i}(z)$ make up the primal and dual lifting stages respectively. The polyphase matrix corresponding to the forward transform is now given by:

$$
\tilde{P}(z)=\left(\begin{array}{cc}
1 / K & 1 \\
1 & K
\end{array}\right) \prod_{i=1}^{m}\left(\begin{array}{cc}
1 & 0 \\
-S_{i}\left(z^{-1}\right) & 1
\end{array}\right)\left(\begin{array}{cc}
1 & -t_{i}\left(z^{-1}\right) \\
0 & 1
\end{array}\right)
$$

In the case of orthogonal filters $\widetilde{P}(z)=P(z)$ many primal and dual lifting steps can exist depending on the factorization. Figure 1 presents the lifting based forward and inverse transform with $\mathrm{m}$ lifting steps.

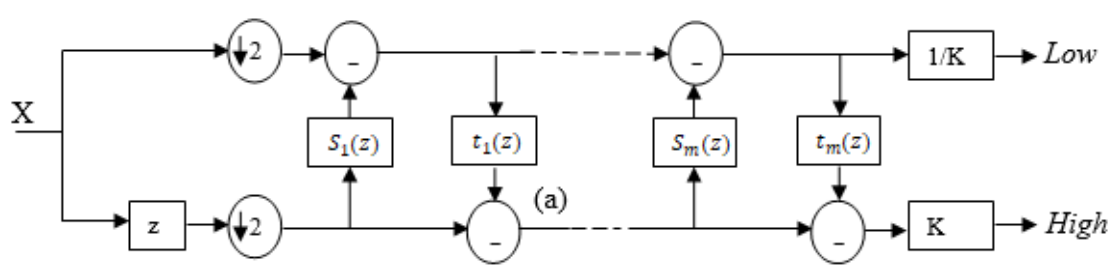

(a)

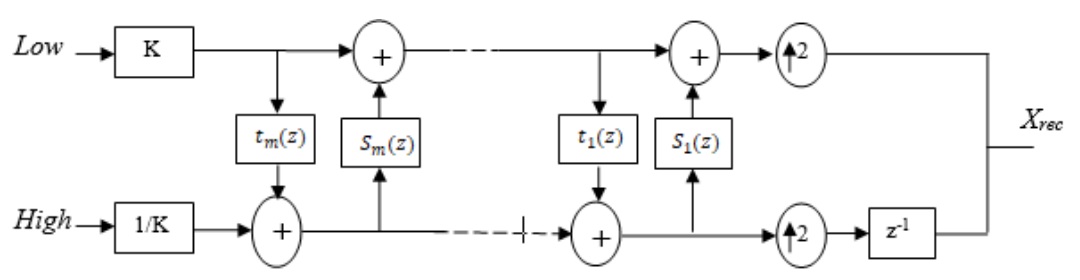

(b)

Figure 1. These figures are; (a) forward transform with $\mathrm{m}$ primal and dual lifting steps, (b) inverse transform with $\mathrm{m}$ primal and dual lifting steps

\section{LIFTING DUAL TREE COMPLEX WAVELET TRANSFORM}

The techniques of lifting are a radically simpler alternative to classic methods for the structure of particular classes of wavelets. there is a good deal of information referring to wavelets easily available from varied sources; many of the newer sources describe the lifting technique for constructing wavelets, it lacks directivity and has solely real coefficients for analysis and processing. Kingsbury [11] formulated the DTCWT to provide near shift invariance and improved directionality with a more compact representation. 
The CWT structure uses a Hilbert transform first applied to the information. The real wavelet transform is then applies to every the original information and also the David Hilbert transformed information, and so the coefficients of every wavelet transform are combined to get a CWT. The DTCWT is a relatively new enhancement to the DWT, with additional properties necessary: it is almost invariant and directionally selective in tow and higher dimensions [5], [12]. These properties underpinned good performance in applications for the image processing (fusion, denoising).

The transform scale in the DTCWT increases, as the size of the subbands decreases in octave steps, in accordance with classic wavelet transformations [13]. This gives each level a trade-off between resolution and redundancy. This does not, however, contribute to a one-to-one relationship across scales between co-located coefficients. Cross-scale relationships are used in fusion, denoising and segmentation applications [13], [14]. In both of those applications the association between a coefficients and its parent is used. Though sub-sampling is reasonable for denoising applications, the DTCWT's subsampled sub-bands have a limited number of coefficients directly linked to each spatial location in the image or signal, a relationship that often contradicts the needs of applications for analysis [15], [16]. To can such analysis we define a lifting form of the DTCWT.

The lifting dual tree complex wavelet transform (LDTCWT), with every sub-band having the same resolution as the signal. It exhibits shift invariance because the LDTCWT includes subsampling (odd and even). It also provides a one-to-one relation between the original samples and each co-located coefficients [17]. The LDTCWT analysis level and synthesis level are shown in Figure 2. Filters at each level are based on the filters used in the CWT.

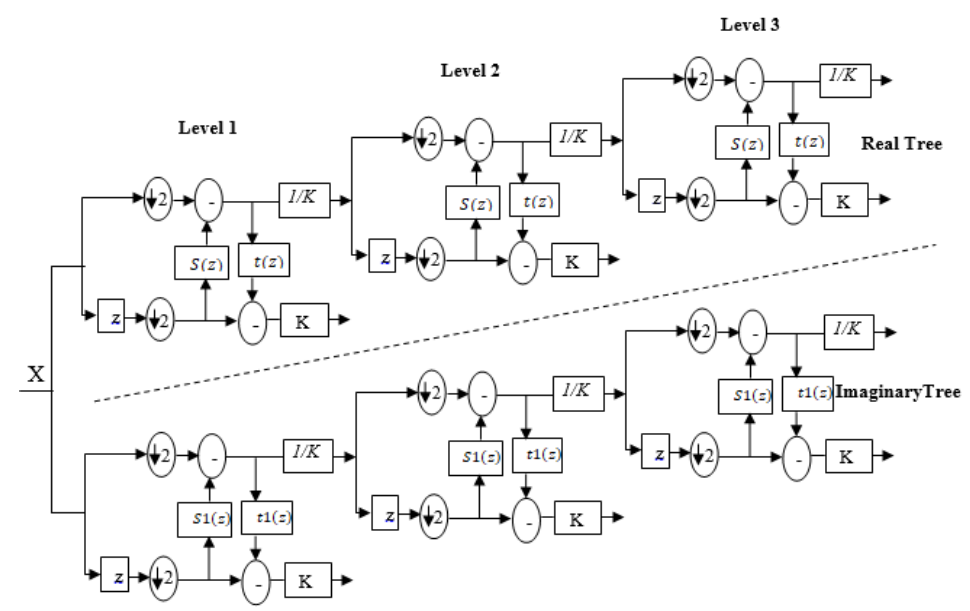

(a)

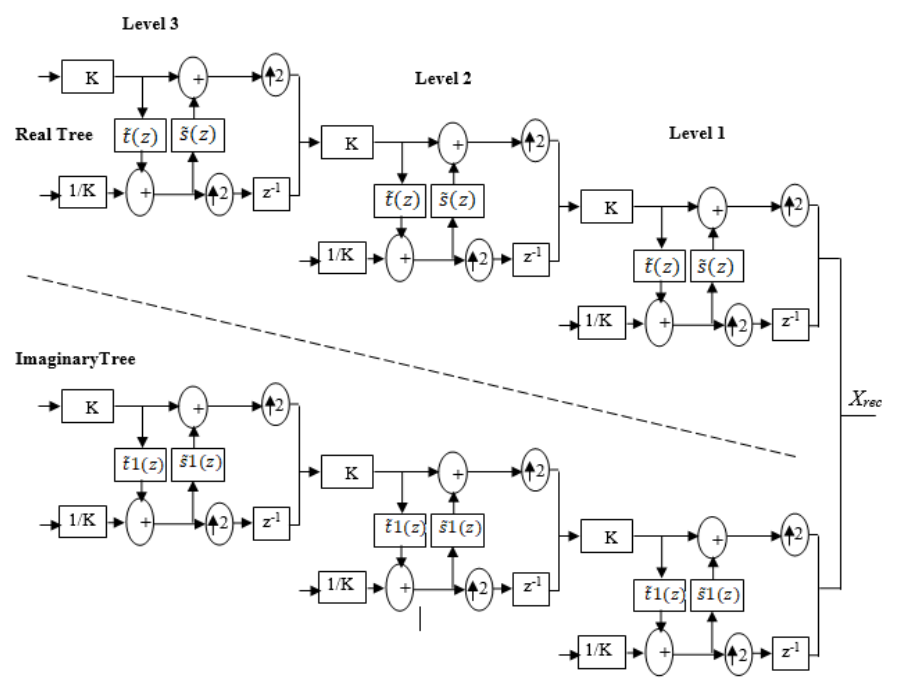

(b)

Figure 2. The LDTCWT analysis level and synthesis level are; (a) analysis for 1-D LDTCWT, (b) synthesis for 1-D LDTCWT 


\section{TOW DIMENSIONAL TRANSFORMS}

The lifting scheme structures for both LDTCWT are identical. Figure 2 shows 1-D analysis and synthesis lifting schemes spanned over three levels. It is evident from the lifting scheme structure of DTDWT that it resembles the lifting scheme structure of LWT with twice the complexity. It can be seen as two LWT trees operating in parallel. One is referred to as a true tree, while the other is referred to as an imaginary tree. The form of conjugate filters used in 1-D LDTCWT is given as (4).

$$
P(x)+j P 1(x)
$$

Where, $\mathrm{P}(\mathrm{x})$ is the set of the primal and dual lifting $\{\mathrm{t}, \mathrm{S}\}$, and $\mathrm{P} 1(\mathrm{x})$ is the set of the primal and dual lifting $\{\mathrm{t} 1, \mathrm{~S} 1\}$ both sets in only $\mathrm{x}$-direction (1-D).

2D structure requires four trees for analysis as well as for synthesis. The pairs of conjugate Filters are applied to two dimensions ( $\mathrm{x}$ and $\mathrm{y}$ ) directions, which can be expressed as (5).

$$
(P(x)+j P 1(x))(P(y)+j P 1(y))=P(x) P(y)-P 1(x) P 1(y)+j(P 1(x) P(y)+P(x) P 1(y))
$$

If we take the real part of this complex wavelet, then we get the aggregate of two separable wavelets.

$$
P(x) P(y)-P 1(x) P 1(y)
$$

The lifting scheme structure of tree $(x) P(y)$, similar to 2-D LWT spanned over 3-level, is shown in Figure 3. All other trees- $P 1(x) P 1(y), P 1(x) P(y)$ and $P(x) P 1(y)$ have identical structures with the appropriate combinations of filters for row-and column-filtering. The overall 2-D dual-tree structure is 4-times redundant than the 2-D LWT. The tree $P(x) P(y)$ and tree $P 1(x) P 1(y)$ form the real pair, while the tree $P 1(x) P(y)$ and tree $P(x) P 1(y)$ form the imaginary pair of the analysis lifting scheme. Trees $\widetilde{P}(x) \tilde{P}(y)$, $\tilde{P} 1(x) \tilde{P} 1(y)$ and trees $\tilde{P} 1(x) \tilde{P}(y), \tilde{P}(x) \tilde{P} 1(y)$ are the real and imaginary pairs respectively in the synthesis lifting scheme similar to their corresponding analysis pairs.

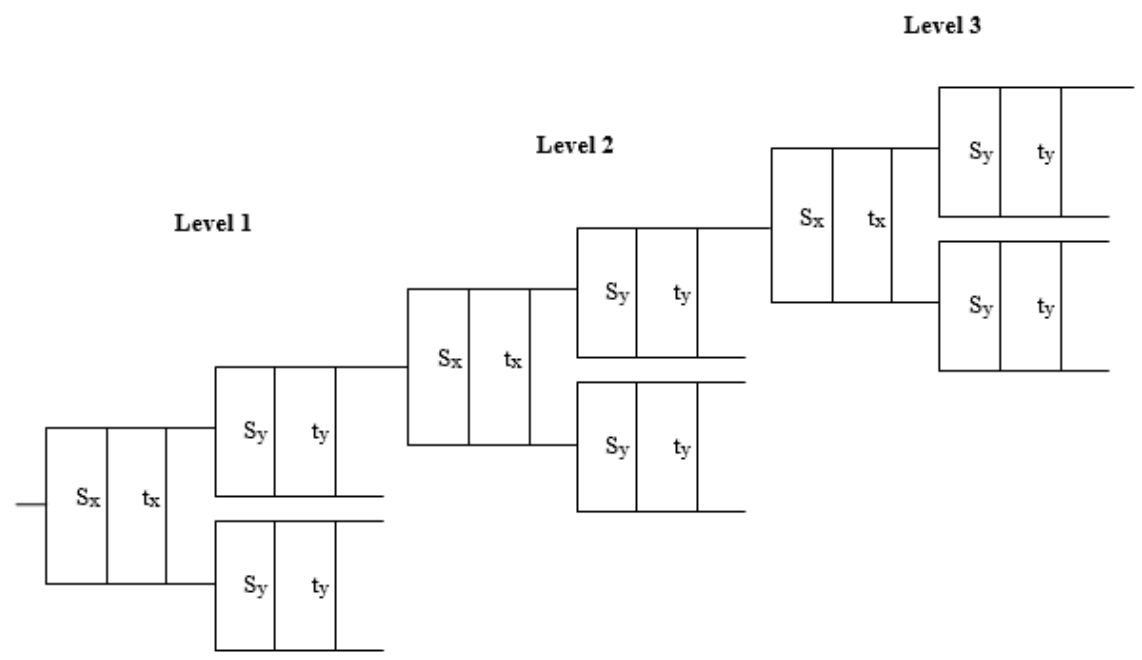

Figure 3. The lifting scheme structure of tree $P(x) P(y)$

\section{APPLICTION: IMAGE DENOISING USING SHARINKAGE FUNCTION}

\subsection{Denoising algorithm}

Wavelet Shrinkage is extremely straightforward nonlinear technique that operates on one wavelet coefficients at a time. In its most simple type, every coefficient is Shrinkage by compare against Shrinkage, if the coefficient is smaller than Shrinkage, set to zero; otherwise it is kept or modified. Reconstruction with the basis signal characteristics and less noise could be achieved by commuting all small noisy coefficients by zero and applying the inverse wavelet transform to the result [5], [18].

Let $s=s_{i, j}, i=\overline{1, M}$ and $j=\overline{1, N}$ is an image of $M \times N$ pixels, which is corrupted by independent and identically distributed (i.i.d.) zero mean, $n_{i, j}$ are independent standard normal $N(0,1)$ random variables 
and $\sigma$ the noise level might be known or unknown [19]. The noise signal can be denoted as $n_{i, j} \sim N\left(0, \sigma^{2}\right)$. This noise might corrupt the signal in a transmission channel. The observed, noise contaminated, image is $x=x_{i, j}, i=\overline{1, M}$ and $j=\overline{1, N}$. Therefore, the noised image can be expressed as (7).

$$
x=s+\sigma n_{i j}
$$

The wavelet coefficients Shrinkage is primarily ruled by either «soft» or «hard» Shrinkage functions as shown in Figure 4. The first function in Figure 4(a) is linear function that does not alter the coefficients and hence is useless for denoising. Within the Figure 4, the linear characteristic is provided only to compare the non-linearity of the two different functions. The hard Shrinkage function is given as (9).

$$
\left\{\begin{array}{l}
z=\operatorname{hard}(\omega)=\omega,|\omega|>\lambda \\
z=\operatorname{hard}(\omega)=0,|\omega| \leq \lambda
\end{array}\right.
$$

Where $\omega$ and $z$ are the wavelets coefficients of the input and output wavelets, respectively. $\lambda$ is a Shrinkage value selected. Similarly, soft thresholding function is given as (9).

$$
\left\{\begin{aligned}
z=\operatorname{soft}(\omega) & =\operatorname{sgn}(\omega) \max (|\omega|-\lambda, 0),|\omega|>\lambda \\
z & =\operatorname{soft}(\omega)=0,|\omega| \leq \lambda
\end{aligned}\right.
$$

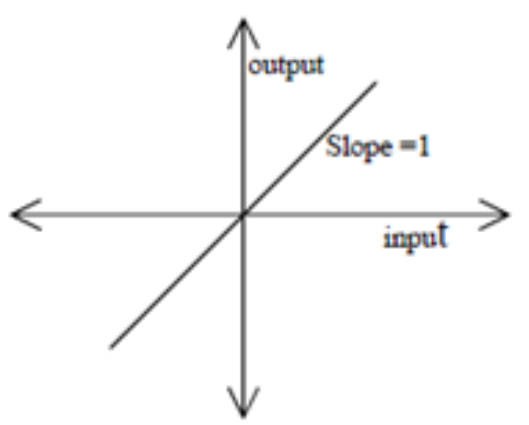

(a)

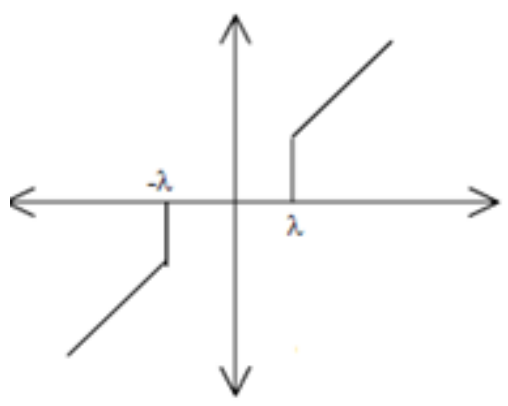

(b)

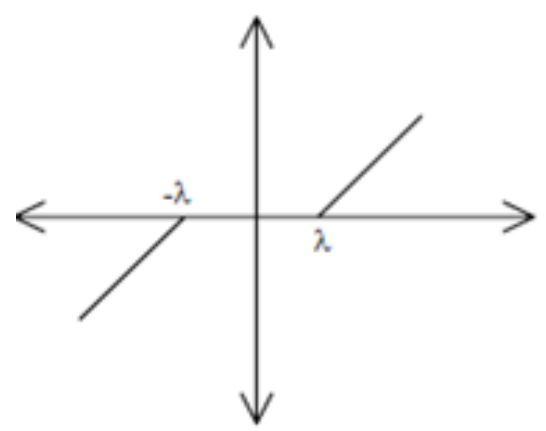

(c)

Figure 4. Shrinkage functions; (a) linear, (b) hard, (c) soft

Shrinkage methods can be grouped into two categories, global Shrinkages and level dependent Shrinkages. The former approach applies a single Shrinkage $\lambda$ value to all empirical wavelets coefficients globally, while the latter method uses different Shrinkages for different levels [5], [20]. We use the universal Shrinkage in this research, which is a simple entropy measure that is proportional to the signal size.

$$
\lambda=\sigma \sqrt{2 \log (k)}
$$

Where $k$ is the size of the signal and $\lambda$ is the Shrinkage value. 


\subsection{Experimental results}

The comparison can be accomplished using mathematical equations to rank each methods performance at a given peak signal to noise ratio (PSNR) [21]. The PSNR has been computed using the formula (10).

$$
P S N R=10 \log _{10}\left(\frac{2^{B}-1}{\sqrt{M S E}}\right)
$$

The structural similarity index measure (SSIM) [15], [22], [23] is a perceptual measure that compares image pixel severity style based on local luminance and pixel disparity. Let $\mathrm{x}$ and $\mathrm{y}$ be two data vectors which should only contain non-negative values and represent the pixel values to be compared with. The mean and standard deviation of $\mathrm{x}$ and $\mathrm{y}$, respectively, estimate the luminance and disparity of those pixels. The SSIM index of $x$ to $y$ is then given by,

$$
\operatorname{SSIM}(x, y)=\frac{\left(2 \mu_{x} \mu_{y}+c_{1}\right)\left(2 \sigma_{x y}+c_{2}\right)}{\left(\mu_{x}^{2}+\mu_{y}{ }^{2}+c_{1}\right)\left(\sigma_{x}^{2}+\sigma_{y}^{2}+c_{2}\right)}
$$

Where;

$$
\begin{array}{ll}
\mu_{x}, \mu_{y} & : \text { Average of } x, \text { average of } y . \\
\sigma_{x}{ }^{2}, \sigma_{y}{ }^{2} & : \text { Variance of } x \text {, variance of } y . \\
c_{1}=\left(k_{1} L\right)^{2}, c_{2}=\left(k_{2} L\right)^{2} & : \text { Two variables to stabilize the division with weak denominator. } \\
\mathrm{L} & \left.: \text { The dynamic range of the pixel-values (typically is } 2^{\text {Bbitsperpixel }}-1\right) . \\
k_{1}=0.01 \text { and } k_{2}=0.03 & \text { (are taken by default). }
\end{array}
$$

The SSIM maps [24], [25] suggest that the proposed approach distributes the image quality more evenly over the image space, and the resulting SSIM index map is perceived as the distorted image quality map. Finally, the quality of all images is determined by using a mean quality map SSIM index. The experiments are conducted on medical image of size $256 \times 256$ at different noise levels $\sigma=5,10,15,20,25$ and 30. To assess the best possible performance of LDTCWT, it is compared with DTCWT. The PSNR and SSIM from various methods are compared in Tables 1 and 2. The LDTCWT outperforms DTCWT most of the time in terms of PSNR and SSIM as well as in terms of visual quality. Moreover LDTCWT is faster than DTCWT. The choice of hard Shrinkage over soft Shrinkage is justified from a soft threshold estimator's results of best possible efficiency.

Table 1. Hard thresholding (PSNR and SSIM for various denoising methods

\begin{tabular}{ccccccc}
\multicolumn{7}{c}{ with parameter 'db8' family wavelets) } \\
\hline$\sigma$ & 5 & 10 & 15 & 20 & 25 & 30 \\
\hline DTCWT & 38.1501 & 38.1315 & 38.1191 & 38.0990 & 38.1351 & 38.0864 \\
& 0.8474 & 0.8469 & 0.8456 & 0.8449 & 0.8447 & 0.8434 \\
LDTCWT & 39.9571 & 39.9407 & 39.9134 & 39.879 & 39.8216 & 39.7643 \\
& 0.8738 & 0.8721 & 0.8685 & 0.8657 & 0.8624 & 0.8563 \\
\hline
\end{tabular}

Table 2. Soft thresholding (PSNR and SSIM for various denoising methods

\begin{tabular}{ccccccc}
\multicolumn{7}{c}{ with parameter 'db8' family wavelets) } \\
\hline$\sigma$ & 5 & 10 & 15 & 20 & 25 & 30 \\
\hline DTCWT & 38.0813 & 38.0898 & 38.0808 & 38.0805 & 38.0550 & 38.0038 \\
& 0.8479 & 0.8472 & 0.8468 & 0.8463 & 0.8460 & 0.8457 \\
LDTCWT & 39.7315 & 39.7061 & 39.6874 & 39.6848 & 39.6565 & 39.5918 \\
& 0.8664 & 0.8635 & 0.8636 & 0.8630 & 0.8610 & 0.8603 \\
\hline
\end{tabular}

To evaluate the subjective effect of LDTCWT and DTCWT, Figure 5 shows the denoising medical images of size $256 \times 256$. It is certain that the image for LDTCWT preserves many local structures best and thus provides the better quality of perceptual image. The visual quality improvement is additionally reflected within the corresponding SSIM map that provides helpful directing on how local image quality is ameliorated over space. In comparison to the SSIM maps for DTCWT, the SSIM map for LDTCWT shows that the areas that are comparatively more organized gain more clearly. The use of larger DWT base functions or wavelets filters causes blurring and ringing noise near edges in images in Figure 5(d), which is one disadvantage of DTCWT. This disadvantage of DWT is overcome in LWT in Figure 5(f). 


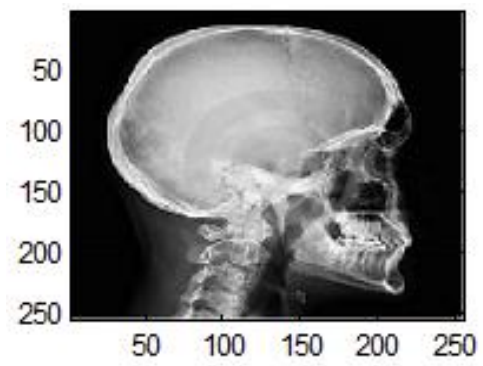

(a)

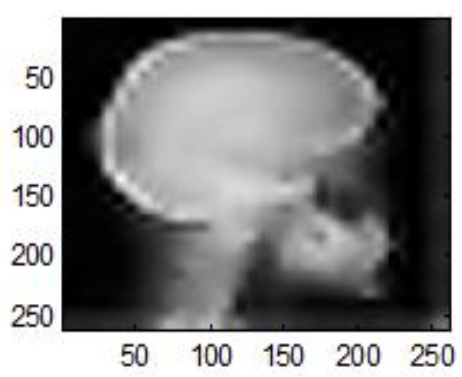

(c)

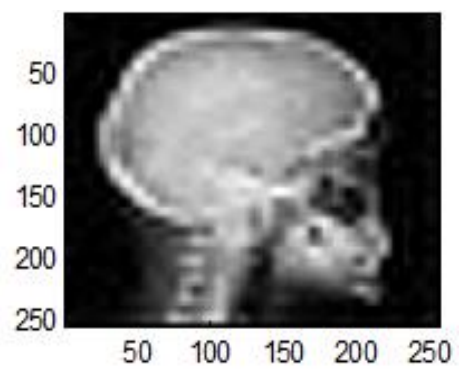

(e)

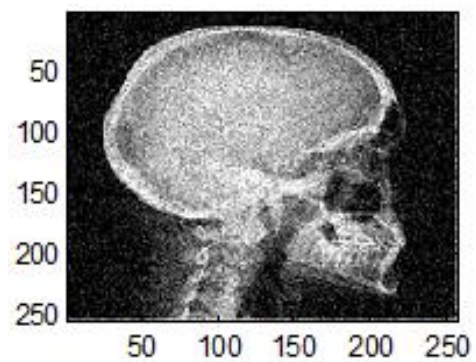

(b)

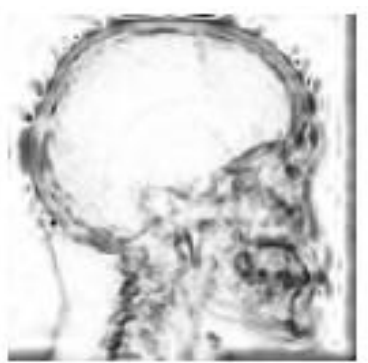

(d)

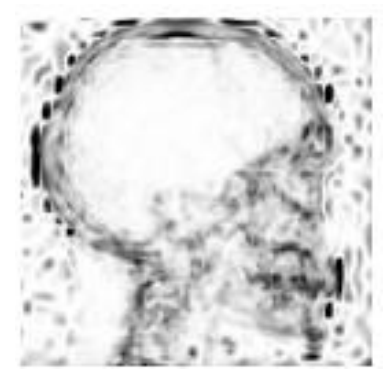

(f)

Figure 5. Evaluation of the subjective effect of LDTCWT and DTCWT, these figures are shows denoising medical images of size $256 \times 256$; (a) original image, (b) noisy image with $\sigma=30$, (c) denoised image using

DTCWT with hard threshold, (d) SSIM map for DTCWT with hard threshold, (e) denoised image with LDTCWT with hard threshold, (f) SSIM map for LDTCWT with hard threshold

\section{CONCLUSION}

We are proposing a new lifting version of the dual tree complex wavelet transform: the LDTCWT. Compared with the Lifting Wavelet transform, the LDTCWT adds improved directionality and phase detail. The LDTCWT also offers an exact invariance compared with the DTCWT, rather than approximate translation invariance. Within a denoising algorithm, all of these attributes are exploited wherever the precise parent of a denoising coefficient is used within a medical image shrinkage system. Firstly, we estimate the noise level of the image. For denoising as a second stage, based on LDTCWT and DTCWT. Using both hard and soft thresholding to shrink wavelet coefficients, their efficiency compared to PSNR (peak signal to noise ratio), SSIM (structural similarity index measure) and SSIM map in image denoising.

\section{REFERENCES}

[1] M. Saeedzarandi, H. Nezamabadi-pour and A. Jamalizadeh, "Dual-Tree complex wavelet coefficient magnitude modeling using scale mixtures of Rayleigh distribution for image denoising," Circuits, Systems, and Signal Processing, vol. 39, no. 8, pp. 2968-2993, 2020, doi: 10.1007/s00034-019-01291-y.

[2] N. Radha and T. R. Babu, "Performance evaluation of quarter shift dual tree complex wavelet transform based multifocus image fusion using fusion rules," International Journal of Electrical \& Computer Engineering (IJECE), vol. 9, no. 4, pp. 2377-2385, 2019, doi: 10.11591/ijece.v9i4.pp2377-2385 .

[3] S. A. Seyyedi, V. Sadau and N. Ivanov, "A Secure Steganography Method Based on Integer Lifting Wavelet Transform,” International Journal of Network Security, vol. 18, no. 1, pp. 124-132, 2016. 
[4] D. B. Taha, T. B. Taha and N. B. Al Dabagh, "A comparison between the performance of DWT and LWT in image watermarking," Bulletin of Electrical Engineering and Informatics (BEEI), vol. 9, no. 3, pp. 1005-1014, 2020, doi: 10.11591/eei.v9i3.1754 .

[5] H. Naimi, A. B. H. Adamou-Mitiche and L. Mitiche, "Medical image denoising using dual tree complex thresholding wavelet transform and Wiener filter," Journal of King Saud University-Computer and Information Sciences, vol. 27, no. 1, pp. 40-45, 2015, doi: 10.1016/j.jksuci.2014.03.015.

[6] S. Wang et al., "Optical image watermarking based on singular value decomposition ghost imaging and lifting wavelet transform," Optics and Lasers in Engineering, vol. 114, pp. 76-82, 2019, doi: 10.1016/j.optlaseng.2018.10.014.

[7] X. Xu, "Single pulse threshold detection method with lifting wavelet denoising based on modified particle swarm optimization," Infrared Physics \& Technology, vol. 88, pp. 174-183, 2018, doi: 10.1016/j.infrared.2017.11.023.

[8] A. K. Singh, "Robust and distortion control dual watermarking in LWT domain using DCT and error correction code for color medical image," Multimedia Tools and Applications, vol. 78, no. 19, pp. 30523-30533, 2019, doi: 10.1007/s11042-018-7115-x.

[9] O. Prakash, C. M. Park, A. Kharef, M. Jeon and J. Gwak, "Multiscale fusion of multimodal medical images using lifting scheme based biorthogonal wavelet transform," Optik, vol. 182, pp. 995-1014, 2019, doi: 10.1016/j.ijleo.2018.12.028.

[10] H. Chen, W. Xu, N. Broderick and J. Han, "An adaptive denoising method for Raman spectroscopy based on lifting wavelet transform," Journal of Raman Spectroscopy, vol. 49, no. 4, pp. 1529-1539, 2018, doi: 10.1002/jrs.5399.

[11] I. W. Selesnick, R. G. Baraniuk and N. C. Kingsbury, "The dual-tree complex wavelet transform," IEEE signal processing magazine, vol. 22, no. 6, pp. 123-151, 2005, doi: 10.1109/MSP.2005.1550194.

[12] N. F. Wang, D. X. Jiang and W. G. Yang, "Dual-tree complex wavelet transform and SVD-based acceleration signals denoising and its application in fault features enhancement for wind turbine," Journal of Vibration Engineering \& Technologies, vol. 7, no. 4, pp. 311-320, 2019, doi: 10.1007/s42417-019-00126-z.

[13] J. D. B. Nelson, A. J. Gibberd, C. Nafornita and N. Kingsbury, "The locally stationary dual-tree complex wavelet model," Statistics and Computing, vol. 28, no. 6, pp. 1139-1154, 2018.

[14] P. R. Hill, N. Anantrasirichai, A. Achim, M. E. Al-Mualla, and D. R. Bull, "Undecimated dual-tree complex wavelet transforms," Signal Processing: Image Communication, vol. 35, pp. 61-70, 2015, doi: 10.1016/j.image.2015.04.010.

[15] L. Mitiche, A. B. Houda Adamou-Mitiche and H. Naimi, "Medical image denoising using dual tree complex thresholding wavelet transform," 2013 IEEE Jordan Conference on Applied Electrical Engineering and Computing Technologies (AEECT), Amman, Jordan, 2013, pp. 1-5, doi: 10.1109/AEECT.2013.6716477.

[16] L. Jing, L. Jingbing, Z. Kun, B. U. Aslam and A. Yang, "Zero-watermarking algorithm for medical images based on dual-tree complex wavelet transform and discrete cosine transform," Journal of Medical Imaging and Health Informatics, vol. 9, no. 1, pp. 188-194, 2019, doi: 10.1166/jmihi.2019.2559.

[17] X. Jin et al., "Infrared and visual image fusion method based on discrete cosine transform and local spatial frequency in discrete stationary wavelet transform domain," Infrared Physics \& Technology, vol. 88, pp. 1-12, 2018, doi: 10.1016/j.infrared.2017.10.004.

[18] Z. Huang, Y. Zhang, Q. Li, T. Zhang and N. Sang, "Spatially adaptive denoising for X-ray cardiovascular angiogram images," Biomedical Signal Processing and Control, no. 40 pp. 131-139, 2018, doi: 10.1016/j.bspc.2017.09.019.

[19] M. Mafi, W. Izquierdo, H. Martin, M. Cabrerizo, M. Adjouadi, "Denoising of ultrasound images affected by combined speckle and Gaussian noise," IET Image Processing, vol. 12, no. 12, pp. 2346-2351, 2018, doi: 10.1049/iet-ipr.2018.5292.

[20] S. Mirghasemi, P. Andreae and M. Zhang, "Domain-independent severely noisy image segmentation via adaptive wavelet shrinkage using particle swarm optimization and fuzzy c-means," Expert Systems with Applications, vol. 133, pp. 126-150, 2019, doi: 10.1016/j.eswa.2019.04.050.

[21] Y. Huang, B. Niu, H. Guan and S. Zhang, "Enhancing image watermarking with adaptive embedding parameter and PSNR guarantee," IEEE Transactions on Multimedia, vol. 21, no. 10, pp. 2447-2460, 2019, doi: 10.1109/TMM.2019.2907475.

[22] P. Etchepareborda, A. Bianchetti, A. L. Vadnjal, A. Federico, "Evaluation of structural similarity quality measures for wrapped recovered phase maps in optical metrology," Optics and Lasers in Engineering, no. 116, pp. 103-110, 2019, doi: 10.1016/j.optlaseng.2019.01.004.

[23] N. R. Hamza, R. A. Dihin and M. H. Abdulameer, "A hybrid image similarity measure based on a new combination of different similarity techniques," International Journal of Electrical \& Computer Engineering (IJECE), vol. 10, no. 2, pp. 1814-1822, 2020, doi: 10.11591/ijece.v10i2.pp1814-1822.

[24] U. Sara, M. Akter and M. S. Uddin, "Image quality assessment through FSIM, SSIM, MSE and PSNR-a comparative study," Journal of Computer and Communications, vol. 7, no. 3, pp. 8-18, 2019, doi: 10.4236/jcc.2019.73002.

[25] J. Peng et al., "Implementation of the structural SIMilarity (SSIM) index as a quantitative evaluation tool for dose distribution error detection," Medical physics, vol. 47, no. 4, pp. 1907-1919, 2020, doi: 10.1002/mp.14010. 